\title{
DESIGN STRATEGY FOR THREE-DIMENSIONAL SUBBAND FILTER BANKS
}

\author{
Po-Cheng Wu, Liang-Gee Chen, Yeong-Kang Lai, and Tsung-Han Tsai \\ Lab. 332, Department of Electrical Engineering \\ National Taiwan University \\ Taipei, Taiwan, R.O.C. \\ E-mail: pcwu@video.ee.ntu.edu.tw
}

\begin{abstract}
Since three-dimensional ( $3-D)$ subband coding has been introduced, most researches on 3-D subband coding perform temporal filtering first. In this paper, however, we investigate the best permutation strategy for temporal, vertical, and horizontal filtering to minimize the requirement of delay elements and find that the results are opposite to our expectation.
\end{abstract}

\section{INTRODUCTION}

Recently, there has been rapid progress in the area of multirate digital signal processing. Applications of multirate systems include subband coding of video and audio signals, fast transforms using digital filter banks, wavelet analysis, and many others [1]-[3]. One of the most important applications of multirate systems is subband coding (SBC). Since it was introduced by Crochiere et al. [4] in 1976, subband coding has been an effective coding approach for video and audio applications [5]-[7]. Subband coding employs a filter bank for splitting the input signal, so the filter banks are essential to subband coding. Because filter banks usually deal with a large number of data, high speed computing hardware is indispensable for subband coding systems.

Subband coding was first extended to three dimensional (temporal, vertical, and horizontal) filtering, i.e., 3-D subband coding, by Karlsson and Vetterli [8]. Since then, most researches which deal with $3-\mathrm{D}$ subband coding perform temporal filtering first, e.g., [8][13]. Since 3-D subband coding requires a large number of delay elements to store the intermediate data, delay elements dominate the hardware cost in this coding scheme. Therefore, the most important task remains how to minimize the requirement of delay elements. In this paper, we investigate the best permutation strategy for temporal, vertical, and horizontal filtering in different conditions in order to minimize the required number of delay elements.

\section{DESIGN STRATEGY}

Let us assume the video format is $M$ lines $\times N$ pixels $\times \mathbf{P}$ frames/sec, and the numbers of filter taps for temporal, vertical, and horizontal filtering are $x+1, y+1$, and $z+1$, respectively. Thus, the required numbers of delay elements for temporal, vertical, and horizontal filtering are $\mathbf{M N x}, \mathbf{N y}$, and $\mathbf{z}$, respectively. In subband coding, we usually employ the FIR direct form filter. The advantage of employing the FIR direct for$m$ is that the lowpass filter and the highpass filter can share the same delay elements.

\subsection{Eight-Full Bands}

We will first consider 3-D subband coding with eightfull bands as shown in Fig. 1. From Fig. 1, we find that by employing the FIR direct form filters, stage 1 requires one set of delay elements; stage 2 requires two sets of delay elements; stage 3 requires four sets of delay elements. The required numbers of delay elements for different permutations of temporal, vertical, and horizontal filtering are listed in Table 1 . Note that the filtering results are identical even though the filtering permutations are different. From Table 1, we find that for eight-full-band splitting, the cascade strategy, which requires the minimum number of delay elements, is horizontal, vertical, followed by temporal filtering, i.e., $(\mathrm{H}, \mathrm{V}, \mathrm{T})$.

\subsection{Four-Reduced Bands}

In order to achieve a high compression ratio in 3-D subband coding, we often discard the four higher bands as shown in Fig. 2. Thus, from Fig. 2, we find that stage 1 requires one set of delay elements; stage 2 requires two sets of delay elements; stage 3 requires three sets of delay elements. The required numbers of delay elements for different permutations are listed in Table 2. From Table 2, we find that for four-reduced-band splitting, the cascade strategy which minimizes the delay 


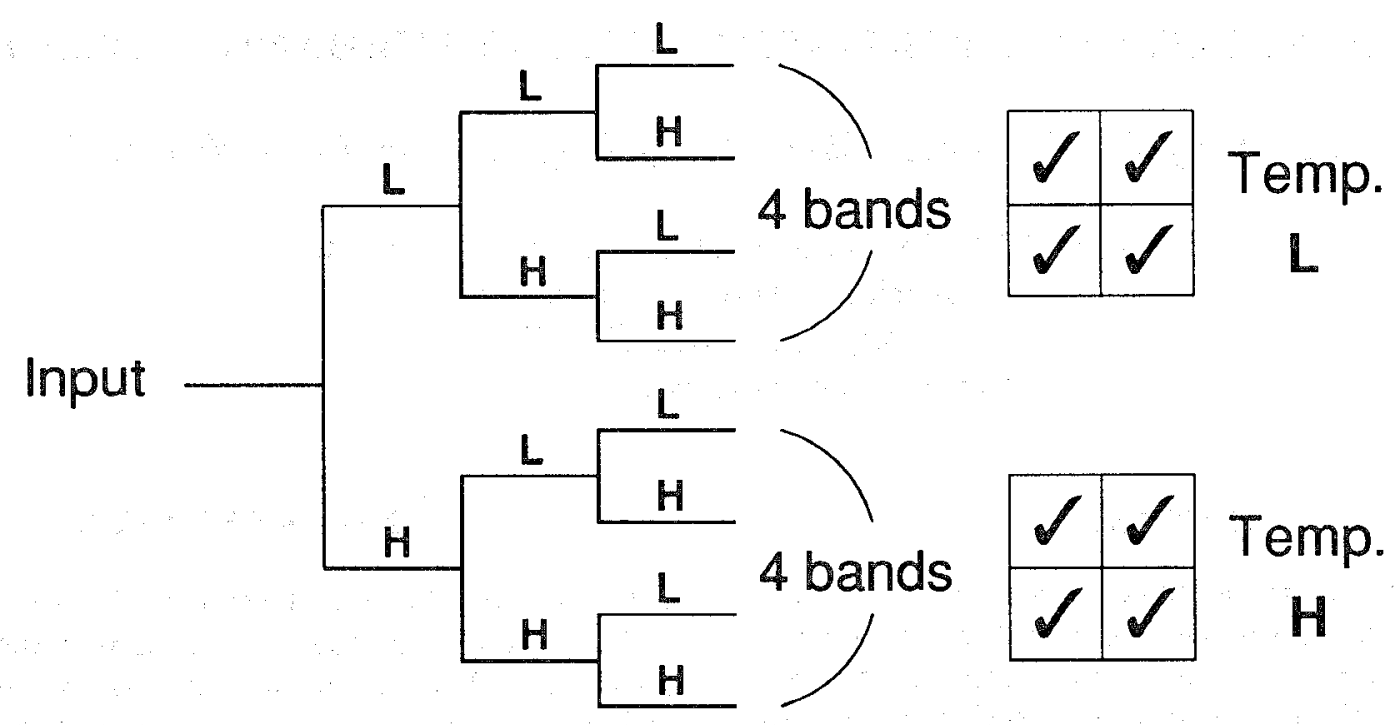

Figure 1: 3-D subband coding with eight-full bands.

\begin{tabular}{|c|c|c||c|c|c|c|}
\hline \multicolumn{2}{|c|}{ Permutations } & Stage 1 $(\times 1)$ & Stage 2 $(\times 2)$ & Stage 3 $(\times 4)$ & Total \\
\hline \hline $\mathrm{T}$ & $\mathrm{V}$ & $\mathrm{H}$ & $M N x$ & $N y$ & $z$ & $M N x+2 N y+4 z$ \\
\hline $\mathrm{T}$ & $\mathrm{H}$ & $\mathrm{V}$ & $M N x$ & $z$ & $N y / 2$ & $M N x+2 N y+2 z$ \\
\hline $\mathrm{V}$ & $\mathrm{T}$ & $\mathrm{H}$ & $N y$ & $M N x / 2$ & $z$ & $M N x+N y+4 z$ \\
\hline $\mathrm{V}$ & $\mathrm{H}$ & $\mathrm{T}$ & $N y$ & $z$ & $M N x / 4$ & $M N x+N y+2 z$ \\
\hline $\mathrm{H}$ & $\mathrm{T}$ & $\mathrm{V}$ & $z$ & $M N x / 2$ & $N y / 2$ & $M N x+2 N y+z$ \\
\hline $\mathrm{H}$ & $\mathrm{V}$ & $\mathrm{T}$ & $z$ & $N y / 2$ & $M N x / 4$ & $M N x+N y+z$ \\
\hline
\end{tabular}

Table 1: Comparison of the required delay elements for different filtering permutations in 3-D subband coding with eight-full bands.

elements is horizontal, vertical, followed by temporal filtering, i.e., $(H, V, T)$.

\subsection{Finite Wordlength Effect}

Finally, if we further consider the finite wordlength effect to enhance the accuracy of subband coding, we assume that the wordlengths of delay elements in stage 1 , stage 2 , and stage 3 are $\mathbf{a}, \mathbf{b}$, and $\mathbf{c}$ bits, respectively. The relation between $\mathbf{a}, \mathbf{b}$, and $\mathbf{c}$ is usually $\mathbf{a} \leq \mathbf{b} \leq \mathbf{c}$. Now, we still look at 3 -D subband coding with four-reduced bands. The required numbers of delay elements for different permutations are listed in Table 3. From Table 3, we are unable to distinguish which permutation strategy is the best choice. Therefore, we make the following assumptions: the video format is $\mathrm{M}=512$ lines and $\mathrm{N}=1024$ pixels; the temporal filter has $\mathbf{x}+1=2$ taps (in order to minimize the delay time); the vertical filter has $y+1=8$ taps; the horizontal filter has $z+1=16$ taps; the wordlengths of delay elements in stage 1 , stage 2 , and stage 3 are $a=8, b=9$, and $c=10$ bits, respectively. After careful calculation, we find that if we consider the finite wordlength effect in the above conditions, the cascade strategy which minimizes the delay elements is vertical, horizontal, followed by temporal filtering, i.e., (V, H, T).

\section{CONCLUSTONS}

From above discussion, we find that the results are opposite to our expectation. Performing the temporal filtering first is not the best strategy. On the contrary, placing the temporal filter at final stage will save the delay elements. We also find that the best permutation strategy for temporal, vertical, and horizontal filtering. depends on different conditions. Finally, for the synthesis filter banks or a higher number of filtering stages in 3-D subband coding, by employing the same scheme, we can also derive the best permutation strategy which minimizes the requirement of delay elements. 


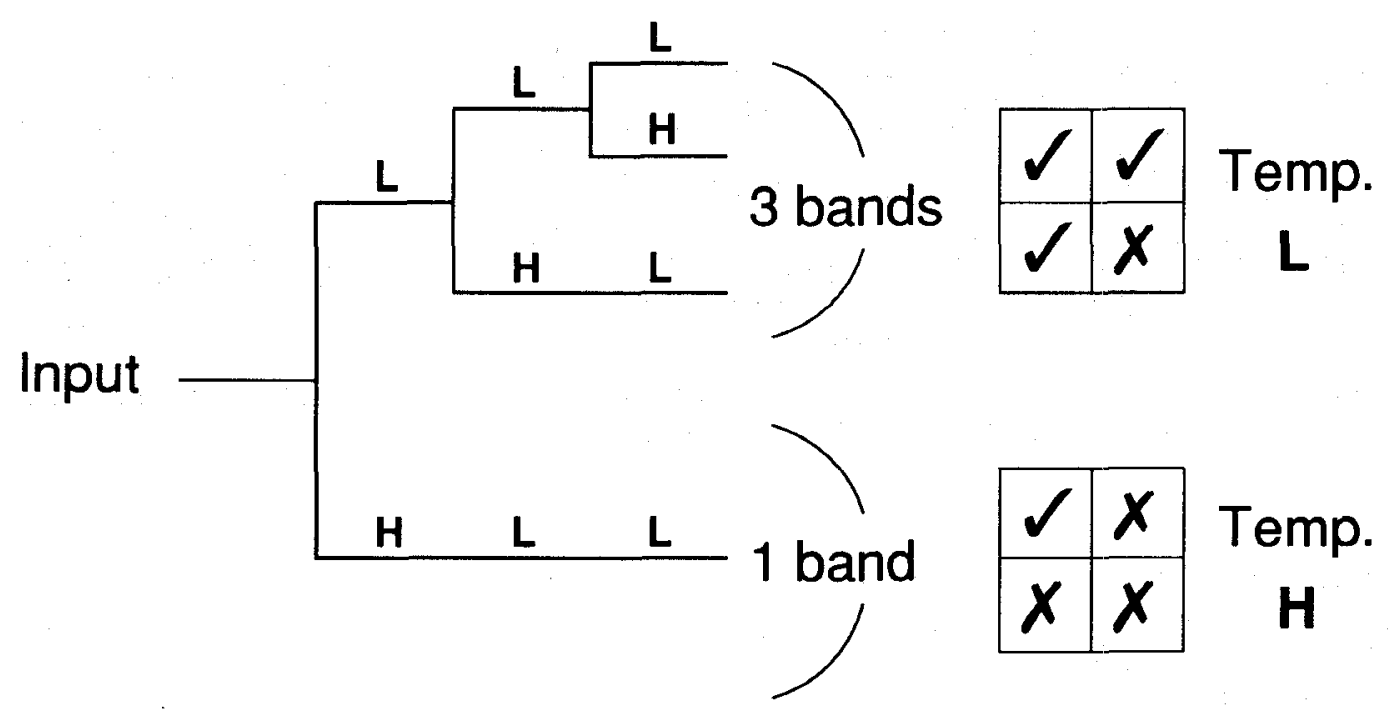

Figure 2: 3-D subband coding with four-reduced bands.

\begin{tabular}{|c|c|c|c|c|c|c|}
\hline \multicolumn{3}{|c|}{ Permutations } & Stage $1(\times 1)$ & Stage $2(\times 2)$ & Stage $3(\times 3)$ & Total \\
\hline$\overline{\mathbf{T}}$ & $\overline{\mathrm{V}}$ & $\mathbf{H}$ & $M N x$ & $\overline{N y}$ & $z$ & $M N x+2 N y+3 z$ \\
\hline $\mathbf{T}$ & $\mathbf{H}$ & $\overline{\mathbf{V}}$ & $M N x$ & $z$ & $N y / 2$ & $M N x+3 N y / 2+2 z$ \\
\hline $\mathbf{V}$ & $\bar{T}$ & $\mathbf{H}$ & $N y$ & $M N x / 2$ & $z$ & $M N x+N y+3 z$ \\
\hline $\mathbf{V}$ & $\mathbf{H}$ & $\mathbf{T}$ & $N y$ & $z$ & $M N x / 4$ & $3 M N x / 4+N y+2 z$ \\
\hline $\mathbf{H}$ & $\mathbf{T}$ & $\mathbf{V}$ & $z$ & $M N x / 2$ & $N y / 2$ & $M N$ 的 $+3 N y / 2+z$ \\
\hline $\mathbf{H}$ & $\overline{\mathbf{V}}$ & $\mathbf{T}$ & $z$ & $N y / 2$ & $M N x / 4$ & $3 \mathrm{MN} x / 4+\mathrm{Ny}+\mathrm{z}$ \\
\hline
\end{tabular}

Table 2: Comparison of the required delay elements for different filtering permutations in 3-D subband coding with four-reduced bands.

\section{REFERENCES}

[1] R. E. Crochiere and L. R. Rabiner, Multirate Digital Signal Processing. Englewood Cliffs, NJ: Prentice Hall, 1983.

[2] P. P. Vaidyanathan, Multirate Systems and Filter Banks. Englewood Cliffs, NJ: Prentice Hall, 1993.

[3] N. J. Fliege, Multirate Digital Signal Processing. New York: Wiley, 1994.

[4] R. E. Crochiere, S. A. Webber, and J. L. Flanagan, "Digital coding of speech in subbands," Bell Syst. Tech. J., vol. 55, pp. 1069-1085, Oct. 1976.

[5] J. W. Woods and S. D. O'Neil, "Subband coding of images," IEEE Trans. Acoust., Speech, Signal Processing, vol. ASSP-34, pp. 1278-1288, Oct. 1986.

[6] H. Gharavi, "Subband coding algorithms for video applications: videophone to HDTV-conferencing,"
IEEE Trans. Circuits Syst. Video Technol., vol.1, no. 2, pp. 174-183, June 1991.

[7] E. B. Richardson and N. S. Jayant, "Subband coding with adaptive prediction for $56 \mathrm{kbits} / \mathrm{s}$ audio," IEEE Trans. Acoust., Speech, Signal Processing, vol. ASSP-34, pp. 691-696, Aug. 1986.

[8] G. Karlsson and M. Vetterli, "Three dimensional sub-band coding of video," in Proc. IEEE Int. Conf. Acoust., Speech, Signal Processing, 1988, pp. $1100-1103$.

[9] J. Hartung, "Architecture for real-time implementation of three-dimensional subband video coding," in Proc. IEEE Int. Symp. Circuits Syst., vol. 3, 1992, pp. 225-228.

[10] K. N. Ngan and W. L. Chooi, "Very low bit rate video coding using $3 \mathrm{D}$ subband approach," IEEE Trans. Circuits Syst. Video Technol., vol. 4, no. 3, pp. 309-316, June 1994. 


\begin{tabular}{|c|c|c||c|c|c|c|}
\hline \multicolumn{3}{|c|}{ Permutations } & Stage 1 $(\times 1)$ & Stage 2 $(\times 2)$ & Stage 3 $(\times 3)$ & Total \\
\hline \hline T & $\mathbf{V}$ & H & $M N x a$ & $N y b$ & $z c$ & $M N x a+2 N y b+3 z c$ \\
\hline T & H & V & $M N x a$ & $z b$ & $N y c / 2$ & $M N x a+3 N y c / 2+2 z b$ \\
\hline V & T & H & $N y a$ & $M N x b / 2$ & $z c$ & $M N x b+N y a+3 z c$ \\
\hline V & H & T & $N y a$ & $z b$ & $M N x c / 4$ & $3 M N x c / 4+N y a+2 z b$ \\
\hline $\mathbf{H}$ & T & V & $z a$ & $M N x b / 2$ & $N y c / 2$ & $M N x b+3 N y c / 2+z a$ \\
\hline $\mathbf{H}$ & V & T & $z a$ & $N y b / 2$ & $M N x c / 4$ & $3 M N x c / 4+N y b+z a$ \\
\hline
\end{tabular}

Table 3: Comparison of the required delay elements for different filtering permutations considering the finite wordlength effect in 3-D subband coding with four-reduced bands.

[11] C. I. Podilchuk, N. S. Jayant, and N. Farvard$\mathrm{m}$, "Three-dimensional subband coding of video," IEEE Trans. Image Processing, vol. 4, no. 2, pp. 125-139, Feb. 1995.

[12] F. Fan, S. Simon, I. Bruyland, W. Zhu, B. D. Canne, and M. V. Bladel, "A method for hierarchical subband HDTV splitting," IEEE Trans. Circuits Syst. Video Technol, vol. 5, no. 3, pp. 225-230, June 1995.

[13] C. H. Chou and C. W. Chen, "A perceptually optimized 3-D subband codec for video communication over wireless channels," IEEE Trans. Circuits Syst. Video Technol., vol. 6, no. 2, pp. 143-156, Apr. 1996. 